\title{
Effect of Hfe Deficiency on Memory Capacity and Motor Coordination after Manganese Exposure by Drinking Water in Mice
}

\author{
Helal Hussain Alsulimani, Qi Ye and Jonghan Kim \\ Department of Pharmaceutical Sciences, Northeastern University, Boston, MA 02115, USA
}

(Received June 17, 2015; Revised August 12, 2015; Accepted September 21, 2015)

\begin{abstract}
Excess manganese (Mn) is neurotoxic. Increased manganese stores in the brain are associated with a number of behavioral problems, including motor dysfunction, memory loss and psychiatric disorders. We previously showed that the transport and neurotoxicity of manganese after intranasal instillation of the metal are altered in Hfe-deficient mice, a mouse model of the iron overload disorder hereditary hemochromatosis $(\mathrm{HH})$. However, it is not fully understood whether loss of Hfe function modifies Mn neurotoxicity after ingestion. To investigate the role of Hfe in oral Mn toxicity, we exposed $H f e$-knockout $\left(H f e^{-t}\right)$ and their control wild-type $\left(\mathrm{Hfe}^{+/+}\right)$mice to $\mathrm{MnCl}_{2}$ in drinking water $(5 \mathrm{mg} / \mathrm{mL})$ for 5 weeks. Motor coordination and spatial memory capacity were determined by the rotarod test and the Barnes maze test, respectively. Brain and liver metal levels were analyzed by inductively coupled plasma mass spectrometry. Compared with the water-drinking group, mice drinking Mn significantly increased Mn concentrations in the liver and brain of both genotypes. Mn exposure decreased iron levels in the liver, but not in the brain. Neither Mn nor Hfe deficiency altered tissue concentrations of copper or zinc. The rotarod test showed that Mn exposure decreased motor skills in $H f e^{+/+}$mice, but not in $H f e^{-t-}$ mice $(\mathrm{p}=0.023)$. In the Barns maze test, latency to find the target hole was not altered in Mn-exposed $H f e^{+/+}$compared with water-drinking $H f e^{+/+}$ mice. However, Mn-exposed $\mathrm{Hfe}^{-/-}$mice spent more time to find the target hole than Mn-drinking $\mathrm{Hfe}^{+/+}$ mice $(p=0.028)$. These data indicate that loss of Hfe function impairs spatial memory upon Mn exposure in drinking water. Our results suggest that individuals with hemochromatosis could be more vulnerable to memory deficits induced by Mn ingestion from our environment. The pathophysiological role of HFE in manganese neurotoxicity should be carefully examined in patients with HFE-associated hemochromatosis and other iron overload disorders.
\end{abstract}

Key words: Barnes maze, Hemochromatosis, Rotarod, Iron overload, Learning, Neurotoxicity

\section{INTRODUCTION}

Manganese $(\mathrm{Mn})$ is an essential metal for normal body function, but is toxic in excess. Exposure to $\mathrm{Mn}$ is associ-

Correspondence to: Jonghan Kim, Northeastern University, Department of Pharmaceutical Sciences, 360 Huntington Avenue 148TF, Boston, MA 02115, USA

E-mail: j.kim@neu.edu

The authors have no conflicting financial interests.

Author contributions: HHA and JK designed research; HHA and QY performed research; HHA and JK analyzed data; HHA and JK wrote the paper.

Abbreviations Used: BBB, blood-brain barrier; Fe, iron; Hfe, High Fe; $\mathrm{HH}$, hereditary hemochromatosis; $\mathrm{Mn}$, manganese.

This is an Open-Access article distributed under the terms of the Creative Commons Attribution Non-Commercial License (http:// creativecommons.org/licenses/by-nc/3.0) which permits unrestricted non-commercial use, distribution, and reproduction in any medium, provided the original work is properly cited. ated with abnormal brain function, including impaired motor coordination, memory deficits and psychiatric disorders, resembling Parkinson's disease (1-3). The neurotoxic effects resulting from $\mathrm{Mn}$ exposure occur in people in industrial settings, such as workers employed in ferroalloy, smelting, mining and welding and agricultural workers exposed to Mn-containing pesticide (4). Mn toxicity is also significant in children living near secondary smelters and in people drinking contaminated water. Moreover, Mn intoxication has been observed in children under long-term parenteral nutrition and patients with chronic liver diseases.

Accumulating evidence has indicated that the absorption of $\mathrm{Mn}$ is altered by body iron status. For example, iron deficient anemia increases intestinal absorption of $\mathrm{Mn}$, whereas iron overload conditions decrease Mn uptake (5). This occurs due to the fact that $\mathrm{Mn}$ is in part absorbed by iron transporters, such as divalent metal transporter 1 (DMT1) and ferroportin, and that the expression of these iron transporters is altered in response to body iron status. Moreover, 
the steady-state concentrations of $\mathrm{Mn}$ in blood are reduced in mice with iron overload, suggesting that elevated iron stores could increase the clearance of Mn (6). Conversely, manganese exposure impairs iron metabolism (7). These results indicate that the transport of iron and manganese is interdependent and further suggest that the neurotoxicity of Mn could be modified by different body iron status.

The question about the mechanistic relationship between iron metabolism and Mn exposure is important because billions of people are affected by a number of iron disorders. In particular, hereditary hemochromatosis $(\mathrm{HH})$ is one of the most common genetic disorders in the Caucasian population. Mutations in HFE ( $\underline{\text { High }} \underline{\mathrm{Fe}}$ ) gene is the major cause of $\mathrm{HH}$, and the two most prevalent HFE missense variants (C282Y and H63D) are found worldwide, including North American (36.9\%), European (34.9\%), African/Middle Eastern (16.7\%) and Asian (2.8\%) populations (8). In normal conditions, HFE controls proper iron metabolism by modulating the production of hepcidin, a 25-amino acid polypeptide hormone produced in the liver and released into blood (9). Hepcidin reduces iron absorption by inducing intracellular degradation of iron transporters, such as ferroportin and DMT1 $(10,11)$. However, hepcidin levels in HFE deficiency are significantly reduced, consistent with elevated expression of iron transporters (12). This is associated with increased iron stores in $\mathrm{HH}$, which promotes a number of disease conditions, including cardiomyopathy, liver cirrhosis, arthritis, diabetes, dyslipidemia and premature death (12-15). Emerging evidence also indicates that $\mathrm{HH}$ is implicated in several neurodegenerative diseases, such as Alzheimer's, Parkinson's and Huntington's diseases.

Our previous study showed that iron deficiency increases olfactory transport of $\mathrm{Mn}$ to the brain and corrects impaired motor function (16). Moreover, recent investigations demonstrated that the transport and neurotoxicity of intranasal Mn are modified in a mouse model of the iron overload disorder hemochromatosis $(2,17,18)$. While these studies have defined the role of HFE in airborne Mn exposure and neurotoxicity, it is largely unknown whether HFE deficiency could modulate the neurotoxic effects of Mn when ingested. In the present investigation, we characterized the effect of HFE on behavioral deficits caused by Mn exposure via drinking water.

\section{MATERIALS AND METHODS}

Ethics statement. Animal studies were conducted in strict accordance with the recommendations from the Guide for the Care and Use of Laboratory Animals of the National Institutes of Health. Animal protocol was approved by the Northeastern University Animal Care and Use Committee.

Animals and Mn exposure. Hfe-knockout $\left(H f e^{--}\right)$and wild-type $\left(H f e^{+/+} ; 129 \mathrm{~S} 6 / \mathrm{SvEvTac}\right.$ background) breeders were kindly provided by Dr. Nancy Andrews (19) (Duke University, NC, USA). Weanling mice were fed facility chow (Prolab Isopro RMH 3000, LabDiet). For the Mntreated group, mice were exposed to a solution of $5 \mathrm{mg}$ $\mathrm{MnCl}_{2} / \mathrm{mL}$ in water ad libitum for 5 weeks. This dose was selected based on the previous study (20). For the control group, mice were allowed to drink water provided by animal facility ad libitum. Water consumption was monitored twice a week and the change in the body weight was measured every week.

Rotarod test. The device consists of a motor-driven knurled cylinder ( $3 \mathrm{~cm}$ in diameter) that is mounted horizontally $24 \mathrm{~cm}$ above a padded surface (Harvard Apparatus, Holliston, MA, USA). Mice were placed on the rotating rod accelerating from 4 to $40 \mathrm{rpm}$ (rotation per minute) over $300 \mathrm{sec}$. Each mouse was subject to three trials per day for four days. Time at which the first loss of footing occurred was recorded. The best score accomplished per each day was used for analysis. Between each trial, the rod was cleaned with Quatricide TB (Pharmacal Research Laboratories Inc., Waterbury, CT, USA).

Barnes maze test. The Barnes maze test was conducted as previously described (18). Briefly, an escape box was placed underneath one of the holes on the maze (Stoelting Co., Wood Dale, IL, USA) and remained the same during the training session for 3 days. Visual cues with different colors and shapes were placed above four quadrants of the maze. In the acclimation period, each mouse was placed by the edge of the escape box. The mouse was gently put into the box if it had not entered the escape box within $1 \mathrm{~min}$. During the training period, the mouse was placed in the center of the maze and covered by an opaque plastic box. After $10 \sim 15 \mathrm{sec}$, the floodlights ( $4 \times 150$ watts) were turned on and the box was then removed. The mouse was allowed to walk around the maze for $3 \mathrm{~min}$ to find and enter the escape box. If the mouse failed to enter the escape box within $3 \mathrm{~min}$, it was gently guided to the box. Three trials were performed per day for 3 days. The latency to enter into the escape box was recorded and analyzed by ANYmaze software (Stoelting Co). On day 4 (test), the escape box was removed and the mouse was allowed to walk around the maze for $90 \mathrm{sec}$ with floodlights on. Time spent to find the target hole where the escape box used to be located (latency) was recorded and analyzed by ANY-maze. The maze was wiped thoroughly with Quatricide TB to avoid olfactory cues.

Tissue collection. After the last behavioral test, mice were euthanized by isoflurane overdose followed by exsanguination. Serum, brain and liver were collected, snap-frozen in liquid nitrogen and stored at $-80^{\circ} \mathrm{C}$ until analysis. 
Non-heme iron assay. The concentrations of non-heme iron in liver samples were analyzed as previously described (21). Briefly, liver samples were digested in a 15 -fold volume of acid solution at $65^{\circ} \mathrm{C}$ for $20 \mathrm{hr}$, followed by the addition of bathophenanthroline disulfonic acid for colorimetric reaction. Liver non-heme iron was calculated as $\mu \mathrm{g} / \mathrm{g}$ liver. Serum iron was determined as previously described with background correction (21) and calculated as $\mu \mathrm{g} / \mathrm{mL}$.

Metal analysis. The concentrations of metals in tissues were determined as previously described (22). Briefly, the left hemisphere of the brain and liver samples were digested in 20\% nitric acid (Trace grade, Fisher Scientific; Pittsburgh, PA, USA) at $125^{\circ} \mathrm{C}$ for $1 \mathrm{hr}$ and diluted to $10 \mathrm{~mL}$ with metal-free double distilled water. Levels of metals were measured by inductively coupled plasma mass spectrometry (ICP-MS) (Varian 810/820MS, Bruker, Billerica, MA, USA). Metal concentrations ( $\mu \mathrm{g} / \mathrm{g}$ tissue) in the samples were determined based on serially-diluted external standards, and all values were normalized using yttrium as an internal standard.

Statistical analysis. Data were presented as means \pm SEM. For two-group comparison (rotarod and Barnes maze tests), the two-sample $t$-test was employed. To determine interaction effects of Hfe deficiency and Mn exposure as well as individual main effects, a two-way ANOVA was performed, followed by post-hoc analysis using SigmaPlot (version 12.3; Systat Software Inc., San Jose, CA, USA).
Differences were considered significant at $p<0.05$.

\section{RESULTS}

Physiological characteristics of $\mathrm{Hfe}^{-1-}$ mice after $\mathbf{M n}$ exposure. Mice exposed to water or to Mn-containing water were euthanized at the age of 8 9 weeks, and physiological values were determined (Fig. 1). Although body weights and water consumption did not differ among the experimental groups, $H f^{-/-}$mice displayed a significant increase in the hematocrit values (Fig. 1C) $(p=0.008)$. Similarly, serum non-heme iron levels were significantly higher in Hfe-deficient mice than in control wild-type mice (Fig. 1D) $(\mathrm{p}=0.002)$. Interestingly, liver non-heme iron levels were altered by both Mn exposure and Hfe gene deficiency. While there was a general increase in liver nonheme iron in $\mathrm{Hfe}^{-/}$mice (Fig. 1E) $(p<0.001)$, Mn exposure significantly reduced liver non-heme iron levels in $H f e^{-/-}$mice $(\mathrm{p}=0.012)$, but not in $H f e^{+/+}$mice.

Effect of Mn exposure on liver metal levels. Levels of metals in the liver were quantified by ICP-MS. Similar to hepatic non-heme iron, total iron levels in the liver were significantly higher in $\mathrm{Hfe}^{-/-}$mice compared with $\mathrm{Hfe}^{+/+}$ mice (Fig. 2) $(p<0.001)$. Mn exposure resulted in a reduction in liver iron levels $(\mathrm{p}=0.044)$, especially in the $\mathrm{Hfe}^{-/-}$ group (Fig. 2) $(\mathrm{p}=0.021)$. As expected, liver Mn levels were increased with Mn exposure in both Hfe-deficient and wild-type mice (Fig. 2) $(p<0.001)$. However, there was no
(A) Body weight

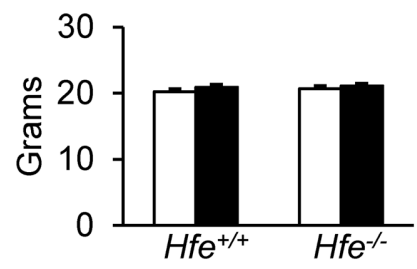

(C) Hematocrit

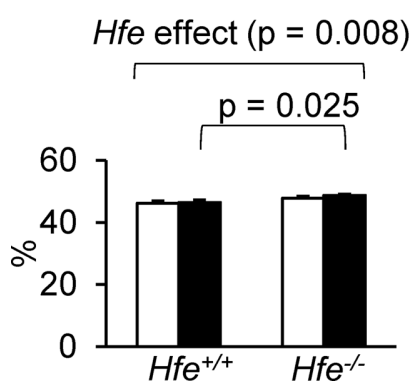

(B) Water consumption

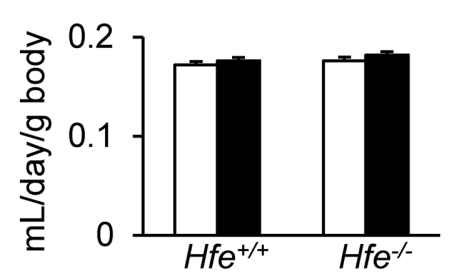

(E) Liver non-heme iron

(D) Serum non-heme iron

Hfe effect $(p=0.002)$

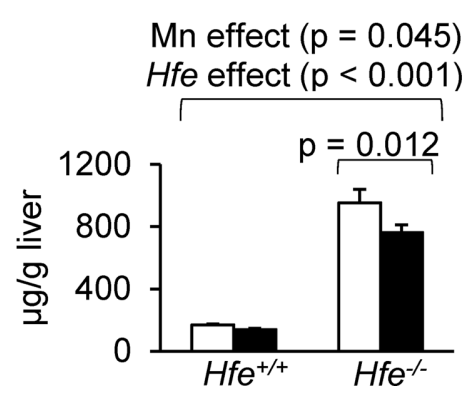

Fig. 1. Effect of $M n$ exposure on physiological characteristics in Hfe-deficient mice. (A) Body weights $(n=19 \sim 24$ per group) of mice 5 weeks post-exposure to $M n$ in drinking water. (B) Mean levels of water consumption ( $n=7 \sim 10$ per group) throughout the exposure period. (C) Hematocrit values ( $n=5 \sim 7$ per group). (D) Non-heme iron levels in serum $(n=5 \sim 7$ per group). (E) Non-heme iron levels in the liver ( $n=13 \sim 20$ per group). Empty and closed bars represent water-drinking and Mn-drinking mice, respectively. Data were presented as means \pm SEM and were analyzed using two-way ANOVA followed by post-hoc comparisons. 


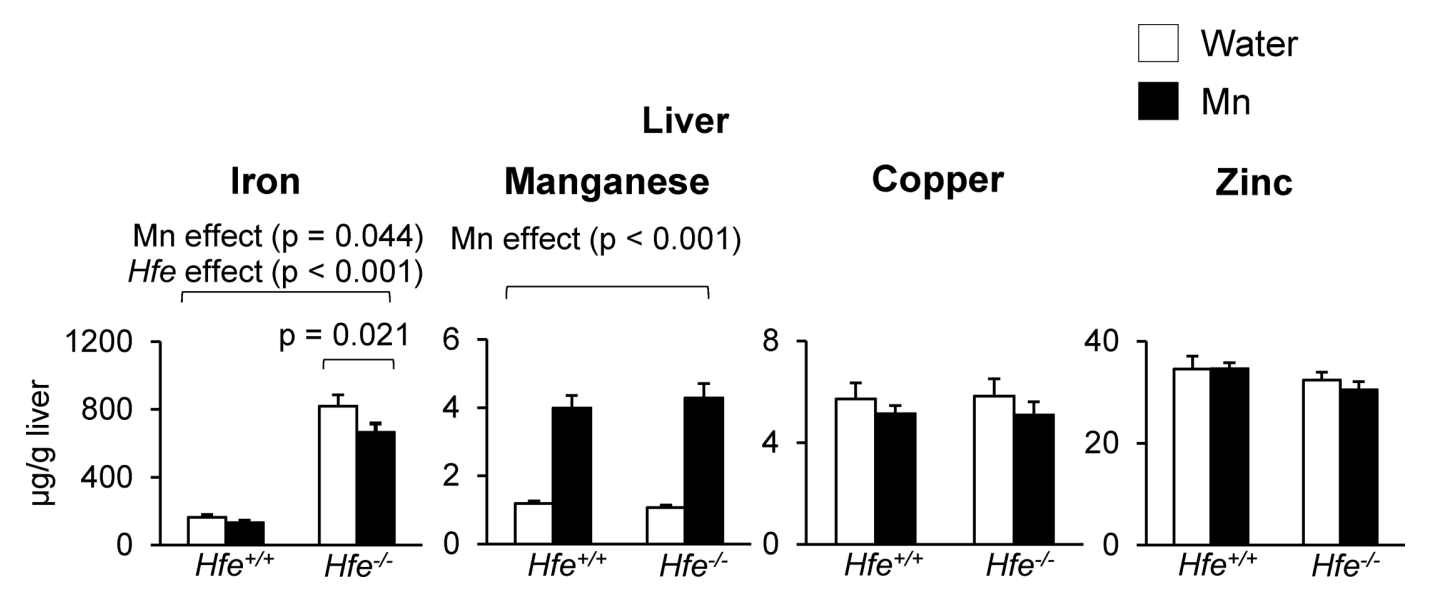

Fig. 2. Liver metal levels after $\mathrm{Mn}$ exposure in drinking water. Levels of iron, manganese, copper and zinc in the liver were measured by inductively coupled plasma mass spectrometry (ICP-MS). Empty and closed bars represent water-drinking and Mn-drinking mice, respectively. Data were presented as means \pm SEM ( $n=6$ per group) and were analyzed using two-way ANOVA followed by post-hoc comparisons.

significant difference in liver Mn levels between $\mathrm{Hfe}^{-/-}$and $H f e^{+/+}$mice after Mn exposure. Neither Mn treatment nor Hfe gene deficiency altered levels of copper or zinc in the liver (Fig. 2).

Effect of Mn exposure on brain metal levels. In contrast to liver iron, brain iron levels were not different among all four groups (Fig. 3). With respect to Mn, basal Mn levels in the brain did not differ between $\mathrm{Hfe}^{+/+}$and $\mathrm{Hfe}^{-/-}$mice. Mn exposure by drinking water elevated brain Mn stores (Fig. 3) $(p<0.001)$ regardless of Hfe expression. Interestingly, there was an interaction effect between Mn exposure and Hfe deficiency on brain $\mathrm{Mn}$; $\mathrm{Mn}$ exposure increased brain Mn levels in $H f e^{+/+}$mice by $81 \%$ compared with those in water-drinking $H f e^{+/+}$mice, whereas Mn-exposed $H \mathrm{fe}^{-/-}$ mice showed a $66 \%$ increase in brain $\mathrm{Mn}$ from the baseline Mn levels observed in water-drinking $\mathrm{Hfe}^{-/-}$mice (Fig. 3)
( $\mathrm{p}=0.047$; Mn x Hfe interaction effect). These results indicate that Hfe deficiency attenuates the distribution of Mn in the brain when $\mathrm{Mn}$ is ingested. Neither Mn exposure nor $H f e$ deficiency altered brain levels of copper or zinc (Fig. 3).

Motor skills in Hfe deficiency after Mn exposure. To examine the effect of Hfe deficiency on motor function upon oral $\mathrm{Mn}$ exposure, the time spent on the rotating rod was measured until the first loss of coordination (Fig. 4). The best performance of each mouse among 3 trials per day on the rod was recorded for 4 days, and the plateau levels of rotarod performance reached on days 3 and 4 (Fig. 4A). Mn-drinking $\mathrm{Hfe}^{-/-}$mice stayed longer on the rod than Mndrinking $H f e^{+/+}$mice $(\mathrm{p}=0.034)$ (Fig. 4A). We then calculated the percentage of the best time over four days between Mn-treated and water-treated mice, and compared these values between $H f e^{+/+}$and $H f e^{-/-}$mice. Mn exposure signifi-

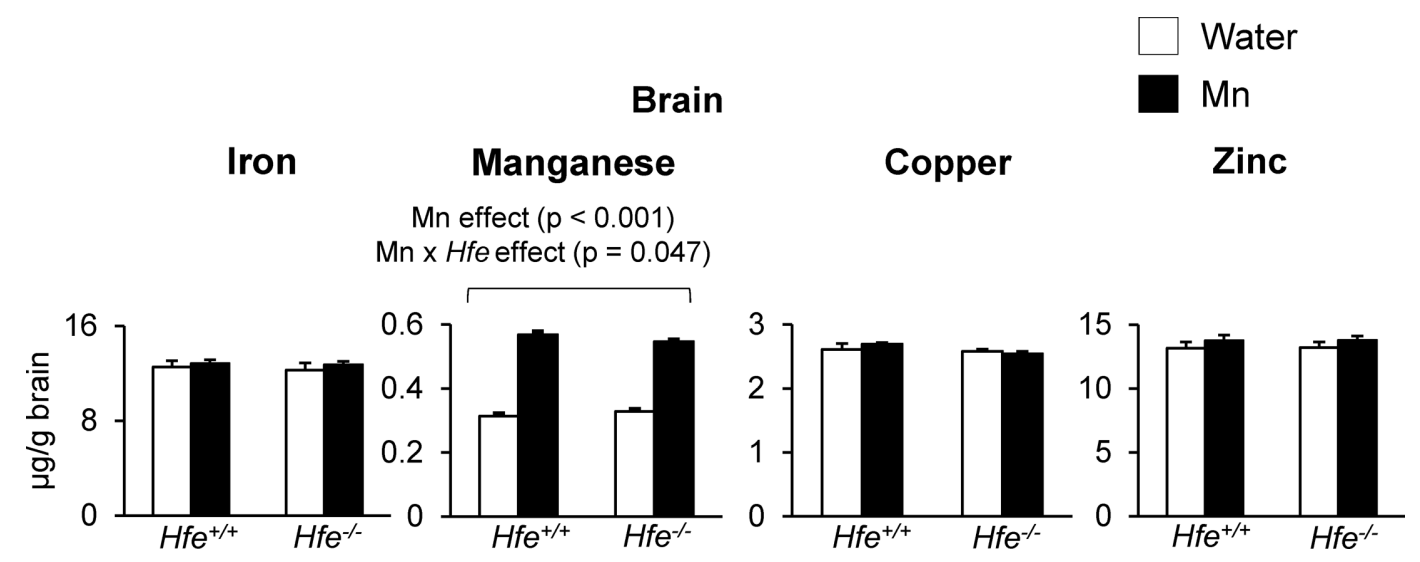

Fig. 3. Brain metal levels after $\mathrm{Mn}$ exposure in drinking water. Levels of iron, manganese, copper and zinc in the brain were measured by ICP-MS. Empty and closed bars represent water-drinking and Mn-drinking mice, respectively. Data were presented as means \pm SEM ( $n=6$ per group) and were analyzed using two-way ANOVA followed by post-hoc comparisons. 
(A) Training

Footing loss

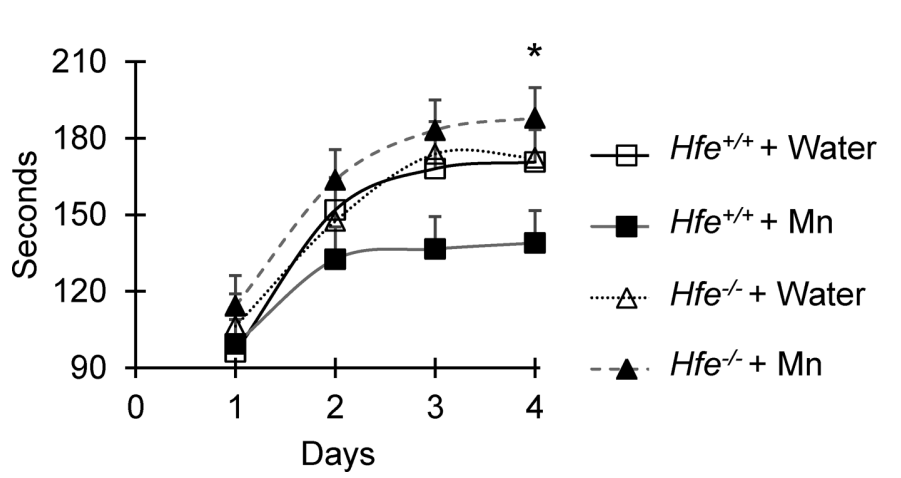

(B) Test

Footing loss

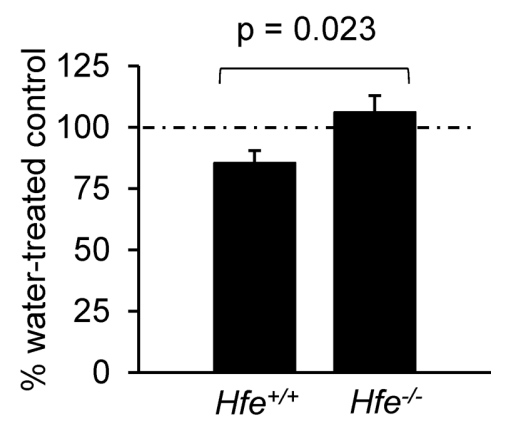

Fig. 4. Effect of $\mathrm{Mn}$ exposure on motor coordination in Hfe-deficient mice. Motor function was determined by the rotarod test. Shown are (A) the time spent before the first footing loss each day over four days among the four groups and (B) the percentage of the best time over four days between Mn-treated mice and water-treated mice. Data were presented as means \pm SEM ( $n=15 \sim 17$ per group) and were analyzed using repeated two-way ANOVA followed by post-hoc comparisons (A) or $t$-test (B). ${ }^{*} p<0.05$ between Mndrinking $\mathrm{Hfe}^{+/+}$and $\mathrm{Hfe}^{-/-}$mice.

cantly reduced motor performance in $\mathrm{Hfe}^{+/+}$mice by $14.7 \%$ (from the baseline performance) (Fig. 4B), but not in $\mathrm{Hfe} e^{-/-}$ mice $(6.1 \%$ increase), indicating an interaction effect between $\mathrm{Mn}$ exposure and Hfe deficiency on motor skills $(\mathrm{p}=0.023)$.

Learning and memory capacity in Hfe deficiency after Mn exposure. To determine if Hfe deficiency modifies the influence of Mn on memory function, Mn-exposed or non-exposed mice were subject to the Barnes maze task (Fig. 5). During training on day $1, \mathrm{Mn}$-exposed $\mathrm{Hfe}^{-/-}$mice spent more time to find the escape box compared either with water-drinking $\mathrm{Hfe}^{-/-}$mice (Fig. 5A) $(\mathrm{p}=0.002)$ or with Mn-exposed $H f e^{+/+}$mice $(p<0.001)$. On days 2 and 3, the time spent to locate the escape box was similar in all four groups of mice (Fig. 5A), suggesting that adequate learning occurred in all groups. On the test day, the time spent to find the target hole was not altered in Mn-exposed $H f e^{+/+}$mice compared with water-drinking $\mathrm{Hfe}^{+/+}$mice (7.1\% reduction) (Fig. 5B). However, Mn-exposed $\mathrm{Hfe}^{-/-}$ mice spent significantly more time to find the target hole than water-drinking $\mathrm{Hfe}^{-/-}$mice did (74.3\% increase) (Fig.
(A) Learning

\section{Latency to the target hole}

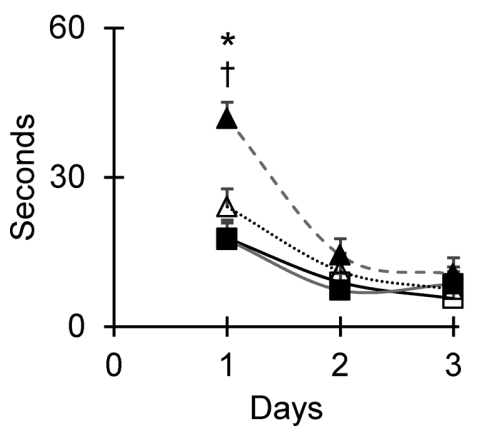

(B) Test

\section{Latency to the target hole}

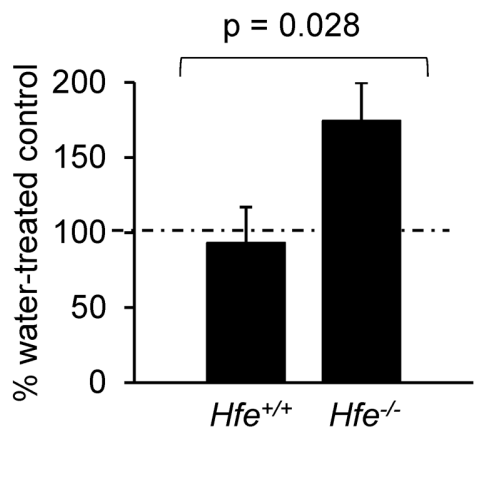

Fig. 5. Effect of Mn exposure on spatial memory capacity in Hfe-deficient mice. Spatial learning and memory capacity were determined by the Barnes maze test. Shown are (A) the latency to the target hole during the learning period over three days of training and (B) the percentage of the latency to the target hole on the test day (day 4) between Mn-treated mice and water-treated mice. Data were presented as means \pm SEM ( $n=15 \sim 17$ per group) and were analyzed using two-way ANOVA followed by post-hoc comparisons (A) or $t$-test (B). ${ }^{*} p<0.05$ between Mn-drinking $\mathrm{Hfe}^{+/+}$and $\mathrm{Hfe}^{-/-}$mice. ${ }^{\dagger} p<0.05$ between water-drinking $H f e^{-/-}$and Mn-drinking $H \mathrm{He}^{-/-}$mice. 
5B), suggesting that loss of Hfe function decreases spatial memory capacity associated with Mn exposure by drinking water $(\mathrm{p}=0.028)$.

\section{DISCUSSION}

Our previous study revealed that neurobehavioral problems after intranasal instillation of $\mathrm{Mn}$ are altered in $\mathrm{Hfe}^{-/}$ mice, indicating that airborne Mn toxicity is modified by the iron overload hemochromatosis $(2,18)$. In the present study, we explored the role of Mn "ingestion" in cognitive and motor functions in $\mathrm{Hfe}^{-/-}$mice. Our results demonstrated that Mn exposure by drinking water decreases motor skills in normal mice, which is corrected by Hfe deficiency. We also showed that loss of Hfe function increases Mn's neurotoxic effect on memory capacity. Since different physiological conditions could affect behavioral performance, we monitored body weights and water intake. There were no changes in body weights among the four groups, suggesting that $5 \mathrm{mg} / \mathrm{mL} \mathrm{MnCl}_{2}$ exposure in drinking water likely has no effect on growth. Water consumption did not differ among all groups, indicating that the total amount of Mn exposure by drinking water was the same between $H f e^{+/+}$and $\mathrm{Hfe}^{-/-}$groups. Our Hfe-deficient mice displayed increased hematocrit, serum iron and liver non-heme iron levels, verifying that loss of Hfe function results in increased body iron stores and represents systemic iron overload conditions $(19,23)$.

In our study, Mn-exposed mice by drinking water had reduced iron levels in the liver. Our results are in agreement with those by Huang et al. (24) who reported a significant decrease in liver iron levels in rats after exposure to $\mathrm{MnCl}_{2}$ by intraperitoneal injection. In addition, we observed that the effect of $\mathrm{Mn}$ on liver iron was more obvious in $\mathrm{Hfe}^{-/-}$ mice than in $\mathrm{Hfe}^{++t}$ mice. This effect could be explained by metal-metal interactions between $\mathrm{Mn}$ and iron in the transport pathway. Rossander-Hulten et al. (25) showed a direct competitive inhibition of $\mathrm{Mn}$ on iron absorption from the gut lumen. Molina et al. (7) also demonstrated that Mn drinking decreases the uptake of iron in the liver after intragastic gavage of ${ }^{59} \mathrm{Fe}$. This effect appears to be specific to $\mathrm{Mn}$ since $\mathrm{Pb}$ drinking did not change ${ }^{59} \mathrm{Fe}$ levels in the liver (7). In contrast to liver iron levels, Mn exposure did not alter brain iron levels. This could result from the difference in the mechanism of iron uptake between the gut lumen and the blood brain barrier (BBB). Iron transport across the BBB is mediated by transferrin and transferrin receptors, and the expression of these proteins in the brain could be regulated upon $\mathrm{Mn}$ exposure to maintain proper levels of brain iron.

One important finding of the current study is that $\mathrm{Hfe}$ deficiency improves impaired motor function caused by Mn exposure. There are mixed results about the role of iron overload in motor skills. Our results are similar to those by
Castro-Caraballo et al. (26) who found an increase in motor function in mice treated with iron dextran. Their mice demonstrated a significant elevation of systemic and liver iron status with no change in brain iron levels, which is similar to our $\mathrm{Hfe}^{-/-}$mice. On the other hand, Golub et al. (27) have shown that Hfe deficiency is associated with movement disorders. Differences in the results of motor test between Golub's and our studies could be due to several different conditions used. First, Golub et al. used C57BL/6J background, whereas in the current study the background strain was 129 S6/SvEvTac. Second, the method of the endpoint measurement was different between the two studies. In Golub's study, a complete falling from the rod was reported, whereas we measured the first loss of footing as an endpoint. Third, Golub reported that Hfe-deficient mice had significantly shorter body length than wild-type mice. Such physical difference could modify the skeletomuscular effect and consequently motor coordination of the mice on the rod.

Mn neurotoxicity is also closely associated with impaired memory function. Several studies reported that Mn exposure causes cognitive deficits and decreased learning ability $(1,18,28)$. Our previous study also showed that intranasal instillation of Mn decreases spatial memory in both $\mathrm{Hfe}^{+/+}$ and $\mathrm{Hfe}^{-/-}$mice (18). Importantly, in the present study we found that $\mathrm{Mn}$ exposure by drinking water impairs spatial memory capacity only in $\mathrm{Hfe}^{-/-}$mice and not in $\mathrm{Hfe}^{+/+}$mice. The difference between the two studies could be due to different doses and duration of exposure and/or routes of exposure to Mn (i.e. intranasal vs. drinking water). It has been recognized that different routes of $\mathrm{Mn}$ administration could alter regional distribution of $\mathrm{Mn}$ in the brain (29). Therefore, it is possible that Hfe deficiency could modify the distribution of Mn within the brain along with the uptake of the metal into the brain through two different pathways, namely, through the air-brain barrier (intranasal) and the BBB (drinking water). Our study also suggests that $\mathrm{Mn}$ exposure at a concentration of $5 \mathrm{mg} / \mathrm{mL}$ in drinking water for 5 weeks does no harm to normal mice, but is sufficient to cause Mn's neurotoxic effects on spatial memory capacity in $\mathrm{Hfe}^{-/-}$mice. A follow-up investigation is warranted to reveal the molecular mechanism(s) of the increased susceptibility to Mn-associated memory deficits in the absence of Hfe.

In addition to exposure-dependent Mn pharmacokinetics discussed above, the effect of $\mathrm{Mn}$ on memory function in $\mathrm{Hfe}^{-/-}$mice could be explained by altered downstream pathways of metal-induced toxicity. Experimental evidence has indicated that the pituitary gland is one of the brain regions that showed a high uptake of Mn (30). As a result, Mn accumulation in the pituitary gland could alter the release of stress hormones, which may be potentiated by increased iron in $\mathrm{Hfe}^{-/-}$mice and consequently impair memory function. Future investigations will address the route-dependent 
accumulation of brain $\mathrm{Mn}$ and region-specific neurochemical alterations under the influence of stress hormones.

In summary, Hfe-deficient mice demonstrated impaired spatial memory when exposed to oral $\mathrm{Mn}$ at the levels where normal mice do not exhibit neurotoxic effects. To our knowledge, this is the first study to examine the neurobehavioral toxicity of oral manganese exposure in Hfe deficiency. Our results suggest that individuals with loss of HFE function could be predisposed to cognitive deficits by prolonged exposure to oral $\mathrm{Mn}$. Since brain metal loading is implicated in the development of neurodegenerative diseases, our findings also suggest that HFE deficiency may be a risk factor for age-related cognitive disorders. Further studies are warranted to address this question and identify novel therapeutic approaches to improve clinical outcomes for memory deficits associated with metal overload in the brain.

\section{ACKNOWLEDGMENTS}

The authors are grateful to Ms. JuOae Chang and Ms. Murui Han for help during animal experiments.

\section{REFERENCES}

1. Cotzias, G.C. (1958) Manganese in health and disease. Physiol. Rev., 38, 503-532.

2. Ye, Q. and Kim, J. (2015) Effect of olfactory manganese exposure on anxiety-related behavior in a mouse model of iron overload hemochromatosis. Environ. Toxicol. Pharmacol., 40, 333-341.

3. Chen, P., Chakraborty, S., Mukhopadhyay, S., Lee, E., Paoliello, M.M., Bowman, A.B. and Aschner, M. (2015) Manganese homeostasis in the nervous system. J. Neurochem., 134, 601-610.

4. Zielhuis, R.L., del Castilho, P., Herber, R.F. and Wibowo, A.A. (1978) Levels of lead and other metals in human blood: suggestive relationships, determining factors. Environ. Health Perspect., 25, 103-109.

5. Davis, C.D., Wolf, T.L. and Greger, J.L. (1992) Varying levels of manganese and iron affect absorption and gut endogenous losses of manganese by rats. J. Nutr., 122, 1300-1308.

6. Claus Henn, B., Kim, J., Wessling-Resnick, M., Téllez-Rojo, M.M., Jayawardene, I., Ettinger, A.S., Hernández-Avila, M., Schwartz, J., Christiani, D.C., Hu, H. and Wright, R.O. (2011) Associations of iron metabolism genes with blood manganese levels: a population-based study with validation data from animal models. Environ. Health, 10, 97.

7. Molina, R.M., Phattanarudee, S., Kim, J., Thompson, K., Wessling-Resnick, M., Maher, T.J. and Brain, J.D. (2011) Ingestion of $\mathrm{Mn}$ and $\mathrm{Pb}$ by rats during and after pregnancy alters iron metabolism and behavior in offspring. Neurotoxicology, 32, 413-422.

8. Hanson, E.H., Imperatore, G. and Burke, W. (2001) HFE gene and hereditary hemochromatosis: a HuGE review. Human Genome Epidemiology. Am. J. Epidemiol., 154, 193-206.

9. Nicolas, G., Bennoun, M., Devaux, I., Beaumont, C., Grand- champ, B., Kahn, A. and Vaulont, S. (2001) Lack of hepcidin gene expression and severe tissue iron overload in upstream stimulatory factor 2 (USF2) knockout mice. Proc. Natl. Acad. Sci. U.S.A., 98, 8780-8785.

10. Nemeth, E., Tuttle, M.S., Powelson, J., Vaughn, M.B., Donovan, A., Ward, D.M., Ganz, T. and Kaplan, J. (2004) Hepcidin regulates cellular iron efflux by binding to ferroportin and inducing its internalization. Science, 306, 2090-2093.

11. Brasse-Lagnel, C., Karim, Z., Letteron, P., Bekri, S., Bado, A. and Beaumont, C. (2011) Intestinal DMT1 cotransporter is down-regulated by hepcidin via proteasome internalization and degradation. Gastroenterology, 140, 1261-1271.

12. Pietrangelo, A. (2010) Hereditary hemochromatosis: pathogenesis, diagnosis, and treatment. Gastroenterology, 139, 393408.

13. Kim, J., Jia, X., Buckett, P.D., Liu, S., Lee, C.H. and Wessling-Resnick, M. (2013) Iron loading impairs lipoprotein lipase activity and promotes hypertriglyceridemia. FASEB J., 27, 1657-1663.

14. Jia, X., Kim, J., Veuthey, T., Lee, C.H. and Wessling-Resnick, M. (2013) Glucose metabolism in the Belgrade rat, a model of iron-loading anemia. Am. J. Physiol. Gastrointest. Liver Physiol., 304, G1095-1102.

15. Kim, J. and Wessling-Resnick, M. (2014) Iron and mechanisms of emotional behavior. J. Nutr. Biochem., 25, 11011107.

16. Kim, J., Li, Y., Buckett, P.D., Böhlke, M., Thompson, K.J., Takahashi, M., Maher, T.J. and Wessling-Resnick, M. (2012) Iron-responsive olfactory uptake of manganese improves motor function deficits associated with iron deficiency. PLoS One, 7, e33533.

17. Kim, J., Buckett, P.D. and Wessling-Resnick, M. (2013) Absorption of Manganese and Iron in a Mouse Model of Hemochromatosis. PLoS One, 8, e64944.

18. Ye, Q. and Kim, J. (2015) Loss of hfe function reverses impaired recognition memory caused by olfactory manganese exposure in mice. Toxicol. Res., 31, 17-23.

19. Levy, J.E., Montross, L.K., Cohen, D.E., Fleming, M.D. and Andrews, N.C. (1999) The C282Y mutation causing hereditary hemochromatosis does not produce a null allele. Blood, 94, 9-11.

20. Kontur, P.J. and Fechter, L.D. (1985) Brain manganese, catecholamine turnover, and the development of startle in rats prenatally exposed to manganese. Teratology, 32, 1-11.

21. Kim, J., Molina, R.M., Donaghey, T.C., Buckett, P.D., Brain, J.D. and Wessling-Resnick, M. (2011) Influence of DMT1 and iron status on inflammatory responses in the lung. Am. J. Physiol. Lung Cell. Mol. Physiol., 300, L659-665.

22. Chang, J., Kueon, C. and Kim, J. (2014) Influence of lead on repetitive behavior and dopamine metabolism in a mouse model of iron overload. Toxicol. Res., 30, 267-276.

23. Chaudhury, C., Kim, J., Mehnaz, S., Wani, M.A., Oberyszyn, T.M., Bronson, C.L., Mohanty, S., Hayton, W.L., Robinson, J.M. and Anderson, C.L. (2006) Accelerated transferrin degradation in HFE-deficient mice is associated with increased transferrin saturation. J. Nutr., 136, 2993-2998.

24. Huang, P., Chen, C., Wang, H., Li, G., Jing, H., Han, Y., Liu, N., Xiao, Y., Yu, Q., Liu, Y., Wang, P., Shi, Z. and Sun, Z. (2011) Manganese effects in the liver following subacute or 
subchronic manganese chloride exposure in rats. Ecotoxicol. Environ. Saf., 74, 615-622.

25. Rossander-Hultén, L., Brune, M., Sandström, B., Lönnerdal, B. and Hallberg, L. (1991) Competitive inhibition of iron absorption by manganese and zinc in humans. Am. J. Clin. Nutr., 54, 152-156.

26. Castro-Caraballo, F., Suárez-Roca, H., Estévez, J. and Bonilla, E. (1992) [Spontaneous motor activity in mice overloaded with iron-dextran]. Invest. Clin., 33, 121-134.

27. Golub, M.S., Germann, S.L., Araiza, R.S., Reader, J.R., Griffey, S.M. and Lloyd, K.C. (2005) Movement disorders in the Hfe knockout mouse. Nutr. Neurosci., 8, 239-244.

28. Guilarte, T.R. and Chen, M.K. (2007) Manganese inhibits
NMDA receptor channel function: implications to psychiatric and cognitive effects. Neurotoxicology, 28, 1147-1152.

29. Roels, H., Meiers, G., Delos, M., Ortega, I., Lauwerys, R., Buchet, J.P. and Lison, D. (1997) Influence of the route of administration and the chemical form $\left(\mathrm{MnCl}_{2}, \mathrm{MnO}_{2}\right)$ on the absorption and cerebral distribution of manganese in rats. Arch. Toxicol., 71, 223-230.

30. Lee, J.H., Silva, A.C., Merkle, H. and Koretsky, A.P. (2005) Manganese-enhanced magnetic resonance imaging of mouse brain after systemic administration of $\mathrm{MnCl}_{2}$ : dose-dependent and temporal evolution of T1 contrast. Magn. Reson. Med., 53, 640-648. 